\title{
Genetic Analysis of Glycoprotein Gene of Indonesian Rabies Virus
}

\author{
Heru Susetya $^{1 *}$, Ito Naoto², Makoto Sugiyama² and Nobuyuki Minamoto² \\ 1) Department of Veterinary Public Health Faculty of Veterinary Medicine, Gadjah Mada University, \\ Yogyakarta, Indonesia \\ 2) United Graduate School of Veterinary Science, Gifu University, Japan
}

\section{Abstract}

The amino acid sequences of the Glycoprotein gene (G gene) of field rabies virus SN01-23 from Indonesia was determined. This isolate showed homology of $93 \%$ in the ectodomain of the Glycoprotein gene to that of the RC-HL strain, which is used for production of animal vaccine in Japan. The high identity in the ectodomain between this field isolate and strain RC-HL suggest that the rabies animal vaccine used in Japan will be effective for rabies street viruses in Indonesia. Result of phylogenetic analysis using the nucleotide sequences of the $G$ genes of rabies street viruses showed that SN01-23 from Indonesia is more closely related to a rabies virus from China than to viruses from Thailand and Malaysia. This genetic data and historical background suggest that rabies viruses in China had been transferred to Indonesia through dogs brought by humans migrating from China to Indonesia.

Keywords : Rabies virus, Glycoprotein gene, Ectodomain, Phylogenetic analysis

\section{Introduction}

Rabies virus belongs to the Lyssavirus genus of the family Rhabdoviridae. This genus is composed of rabies virus (genotype 1) and rabies-related viruses, including Lagos bat virus (genotype 2), Mokola virus (genotype 3), Duvenhage virus (genotype 4), European bat lyssaviruses 1 and 2 (genotypes 5 and 6, respectively), and Australian bat lyssaviruses (genotype 7) (Gould et al., 1998). The virus has a singlestranded RNA genome of approximately 12 $\mathrm{kb}$ containing coding information for nucleocapsid $(\mathrm{N})$, phosphoprotein, matrix protein, glycoprotein and RNA polymerase protein with a negative sense.

The $G$ protein of rabies virus is involved in the induction of virus-neutralizing production and protection after vaccination (Wiktor et al., 1973; Luo et al., 1998). The G

*Corresponding author: Heru Susetya, Department of Veterinary Public Health, Faculty of Veterinary Medicine, Gadjah Mada University, Jl. Olah Raga Karangmalang, Yogyakarta 55281, Indonesia. Tel: 62274-649-62307; E-mail: herususetya@ugm.ac.id protein has also been suggested to be the major factor contributing to the pathogenecity of rabies virus (Morimoto et al.,1999). Therefore, analysis of the G protein is important to understand the antigenicity and pathogenicity of rabies virus. However, there is limited information on the $G$ gene and protein of rabies street viruses in Southeast Asia especially in Indonesia.

As described in this paper, the $G$ gene from Northern Sumatra in Indonesia was analyzed and the sequence was compared with those of vaccine strains. Phylogenetic analysis was also carried out to estimate the genetic relationships between these viruses and other street viruses in the world.

\section{Materials and Methods \\ Sample}

Brain sample from dog that had been diagnosed as having rabies, was obtained from Northern Sumatra of Indonesia.

\section{RT-PCR}

Total RNA was extracted from the brain specimens using a Mag Extractor RNA Kit 
(TOYOBO, Japan). Complementary DNA (cDNA) of the rabies genome was synthesized using Ready-To-Go You-Prime First-Strand Beads (Amersham Pharmacia Biosciences, U.S.A) with the antigenomic sense primer RHN17-2 (5'-TTCAAAGTCAA TAATCAGGTGG-3'), which corresponds to nucleotides at positions 92 to 113 of genome. In the first step, the cDNA was amplified by PCR using PCR Beads (Amersham Pharmacia Biosciences) with the antigenomic sense primer RHM-A2 ( 5 ' A C A A T G T C A T A T C C AAGGCAGAATCTGGTG-3') acting at positions 2976 to 3005 and the genomic sense primer RHL-A2 (5'AGAGTTCCT CAAGATGTTGGGGACAATGGG-3') acting at positions 5458 to 5487. PCR was performed in a TaKaRa PCR Thermal Cycler MP (TAKARA, Japan) in $25 \mu$ l of reaction mixture. The profiles of first and second PCR consist of an initial denaturation for $5 \mathrm{~min}$ at $95^{\circ} \mathrm{C}$, followed by 40 cycles of denaturation for $30 \mathrm{~s}$ at $95^{\circ} \mathrm{C}$, reannealing for $1 \mathrm{~min}$ at $60^{\circ} \mathrm{C}$, and elongation for $2 \mathrm{~min}$ at $72^{\circ} \mathrm{C}$, and a final incubation for $5 \mathrm{~min}$ at $72^{\circ} \mathrm{C}$. The second PCR was performed using the antigenomic sense primer RGP-A1 (5'AACATCC CTCAAAAGACTTAAGGA AAGATG-3') acting at positions 3290 to 3319 and the genomic sense primer RHL-1 (5'CCTAGGTCTCCAGGATCGAGCAT-3') acting at positions 5412 to 5434, resulting in a 2145 bp DNA fragment.

\section{Direct sequencing and genetic analysis}

Cy5-labeled antigenomic sense primers IGSEQ2F (5'-TGTGGATTTGTGGA TGAAAGAGGC-3') at positions 3993 to 4016 and RGSEQ2F (5'-CTCAGGGGTT GACCTG GGTCTCCC-3') at positions 4655 to 4678 and genomic sense primers IGSEQ1R (5'GGCTCTGCATGCATCTGG CGTCGG-3') at positions 3639 to 3662, RGSEQ7R (5'TAAGTCTAAGTCCAAGAA CTCCAC-3') at positions 4056 to 4079 and IGSEQ3R (5'-
CTGCTCTATTAGCTTTTCT GCAGC-3') at positions 4753 to 4776 were employed for sequencing of the amplified cDNA. After purification of the second PCR product using an UltraClean Kit (MO BIO, U.S.A), a cycle sequencing reaction was carried out using an AutoCycle Sequencing Kit (Amersham Pharmacia Biosciences). Sequencing product was analyzed using an ALF DNA Sequencer (Amersham Pharmacia Biosciences). The calculation of homology and alignment of deduced amino acid sequences were carried out using Genetyx software for Macintosh, version 6.0.1. Rabies isolates from Thailand and Indonesia were compared phylogenetically with those of Lyssaviruses in the world, information being obtained from the Gene Bank database. Phylogenetic analysis was performed by the neighborjoining method (Saitou and Nei, 1987) using Clustal X. The bootstrap probabilities (BSPs) were calculated using 1000 replicates.

\section{Results}

The $G$ gene rabies virus SN01-23, in Indonesia was found to have an open reading frame of 1575 nucleotides in length coding for 524 amino acids (Figure 1). The nucleotide sequences of this rabies virus exhibit high levels of homologies $(95.7 \%$ to $97.6 \%)$ to other. The derived $G$ protein was found to be composed of a signal peptide (19 amino acids), an ectodomain (amino acids 1-439) protruding from the viral membrane, a transmembrane domain (amino acids 440461), and a cytoplasmic domain (amino acids 462-505), which interacts with internal proteins (Mebatsion et al., 1999) as has been found for other rabies viruses.

The deduced amino acid sequences of the $\mathrm{G}$ was compared with that of the RC-HL strain (Ito et al., 1994), a virus strain used for production of animal vaccine in Japan (Figure 1 ). The amino acid identities between SN01-23 and RC-HL strains were 


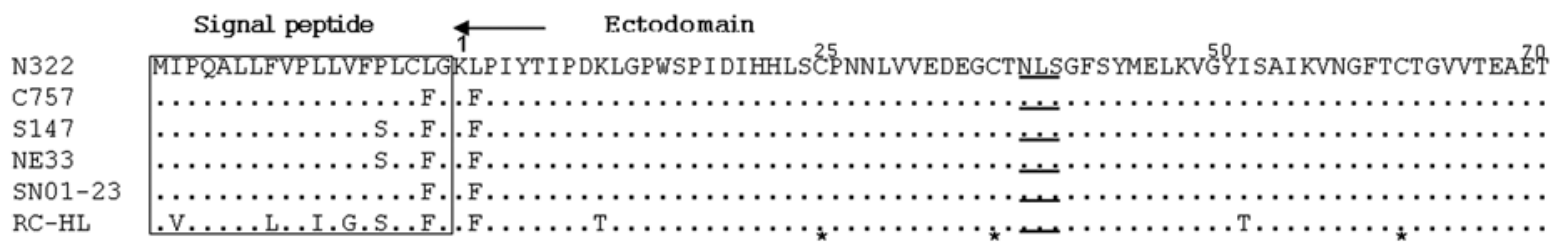

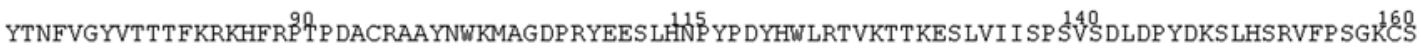

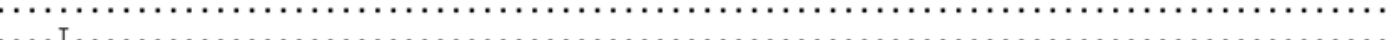
 $\mathrm{RC}-\mathrm{HL}$

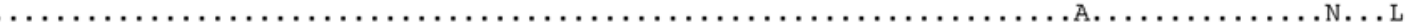

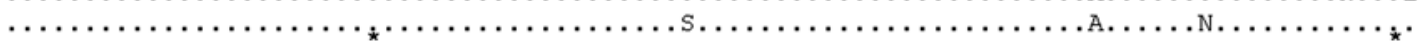

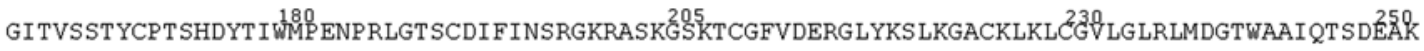

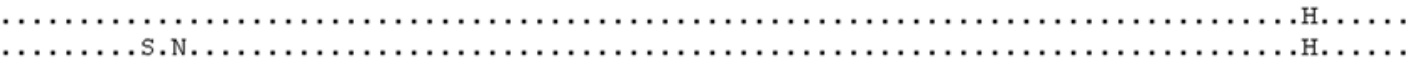

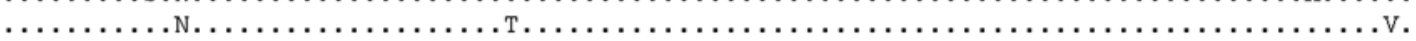

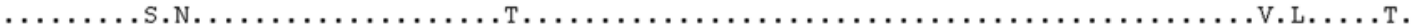

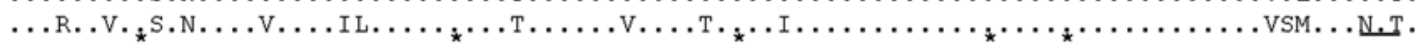

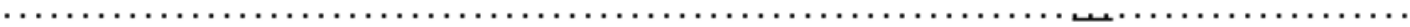

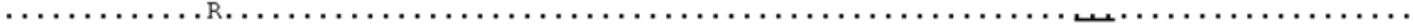
n.m.

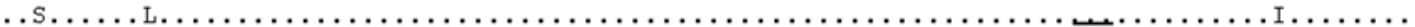
$\mathrm{RC}-\mathrm{HL}$

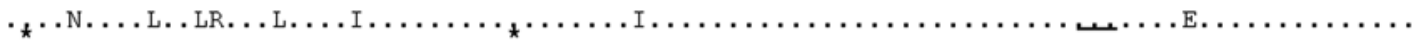

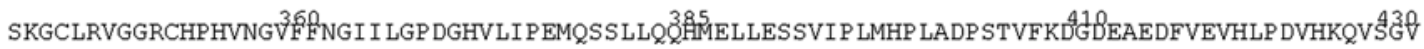

\begin{tabular}{|c|c|}
\hline Ectodom & $\longrightarrow$ Trar \\
\hline N322 & GKYYVLMSVGALIALMLI \\
\hline C757 & $\ldots \ldots$ T..... \\
\hline S147 & $\ldots \ldots$ A.LI $\ldots$ G........ \\
\hline NE33 & ........ I. \\
\hline SN01-23 & .RA..I..S.G...... \\
\hline $\mathrm{RC}-\mathrm{HL}$ & 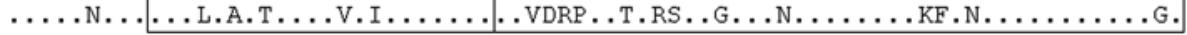 \\
\hline
\end{tabular}

Figure. 1. Alignment of the deduced amino acid sequences of the rabies $\mathrm{G}$ protein from field viruses in Indonesia, Thailand and from the RC-HL strain. Asterisks indicate cysteines. Boxes show the signal peptide, transmembrane domain, and cytoplasmic domain. Underlined motifs are potential N-glycosylation sites. 


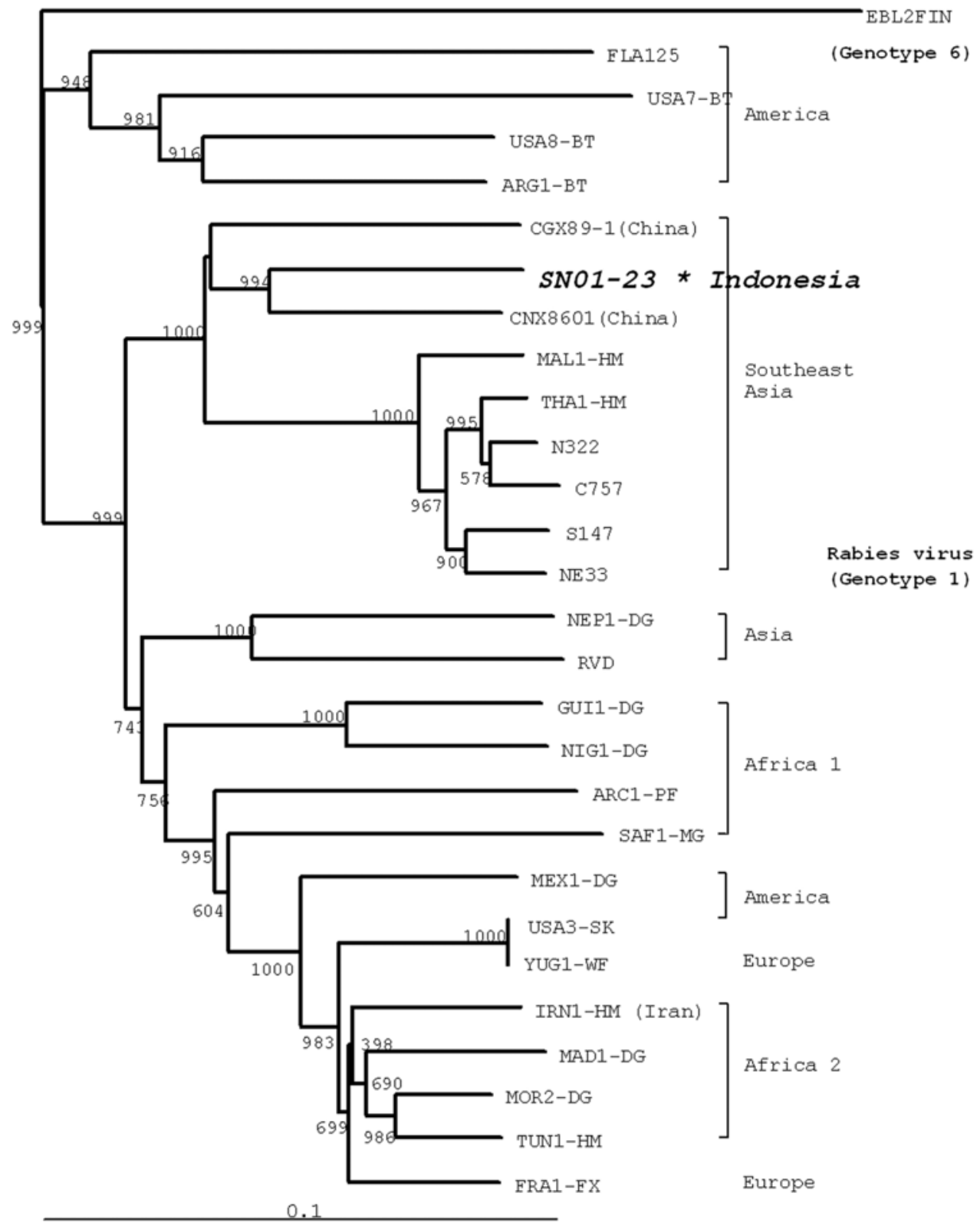

Figure. 2. Phylogenetic tree of rabies street viruses. The tree was constructed using the neighbor-joining method on the basis of nucleotide sequences of the $\mathrm{G}$ gene. Bootstrap values of 1000 replicates indicate the robustness of the corresponding nodes. Horizontal lines indicate nucleotide differences. 
$88.5 \%, 73.7 \%, 92.0 \%, 68.2 \%$ and $68.2 \%$ in the overall G protein, signal peptide, ectodomain, transmembrane domain and cytoplasmic domain respectively. A rabies isolate in Indonesia showed 94.3-95.4\%, 94.7$100 \%, 96.0-97.0 \%, 72.7-81.8 \%$ and $84.1-90.2 \%$ in amino acid homologies in the overall G protein, signal peptide, ectodomain, transmembrane domain and cytoplasmic domain, respectively, to those of isolates in Thailand.

All 14 cysteines in the ectodomain are completely conserved (Figure 1). The Indonesia isolate has two potential N-linked glycosylation sites at amino acids 37 and 319. These sites of the $G$ protein are conserved.

Phylogenetic analysis based on nucleotide sequences of the $G$ genes of the Indonesia and rabies viruses from various parts of the world revealed that rabies isolate of Indonesia form one cluster as a Southeast Asian lineage, which is supported by the high bootstrap probability of 1000 (Figure 2). A rabies isolate in Indonesia is more closely related to a strain virus from China than to viruses from Thailand and Malaysia.

\section{Discussion}

The ectodomain is the most conserved part of the $G$ protein as described in Lyssaviruses (Badrane and Tordo, 2001). It has been reported that two Lyssaviruses will cross-neutralize each other if they have more than $72 \%$ amino acid identity in the ectodomain (Badrane et al., 2001). The high identities of $92-93 \%$ in the ectodomain between all isolates analyzed in this study and the RC-HL strain suggest that the rabies animal vaccine used in Japan will be effective for field viruses in Thailand and Indonesia. Other rabies vaccines, which are prepared by using strains PV, SAD B19, ERA and HEPFlury, are also expected to be effective in Thailand and Indonesia, because there are high identities of $94-96 \%$ in the ectodomain between these isolates and the vaccine strains.

All 14 cysteines in the ectodomain is completely conserved, suggesting that structure of the ectodomain is similar to those of other Lyssaviruses (Walker and Kongsuwan, 1999). There are two potential $\mathrm{N}$-linked glycosylation sites, including a glycosylation site at amino acid positions 319, that are conserved among all Lyssaviruses (Badrane et al., 2001; Walker and Kongsuwan, 1999)

This findings show the importance of the structural and functional constraints on the ectodomain in contrast to other parts of the $\mathrm{G}$ protein and the considerable genetic diversity of rabies viruses. Therefore, nucleotide sequencing data of the $G$ protein genes of twenty-three rabies street viruses from other countries were used for determination of genetic relationships with the field rabies virus from Indonesia. Figure 2 shows a phylogenetic tree based on nucleotide sequences of the $G$ genes of rabies street viruses and an EBL2 virus as an outgroup. Rabies street viruses from Southeast Asia (Malaysia, Thailand and Indonesia) and China form a genetic cluster in this phylogenetic tree. This genetic relationship is supported strongly by the high BSP of 1000. Interestingly, SN01-23 rabies virus from Indonesia is more closely related to strain CNX8601 from Ningxia in the Northwest part of China (Tang et al., 2000) than to rabies viruses from Malaysia and Thailand, which are in geographical proximity to Indonesia. Results of phylogenetic analysis using nucleoprotein gene sequences also showed that the field rabies viruses from Indonesia are genetically closer to a virus from China than to viruses from Thailand (data not shown). These data obtained by phylogenetic analysis using the $G$ gene support the assumption that rabies street viruses in China had been transferred to Indonesia through dogs brought by 
humans who had migrated from China to Indonesia.

Further studies on other field rabies viruses from other area of Indonesia and in countries located between Indonesia and China are needed to elucidate the dynamics of rabies viruses in Southeast Asia. Such a genetic database would be useful for tracing the routes of rabies infection and for establishing measures to eliminate rabies viruses in Southeast Asia.

\section{Acknowledgment}

This study was supported in part by Grand-in-Aid for Scientific Research from Ministry of Education, Culture, Sports, Science and Technology Japan.

\section{References}

Badrane, H., Bahloul, C., Perrin, P. and Tordo, N., 2001. Evidence of two Lyssavirus phylogroups with distinct patogenicity. J. Virol., 75, 3268-3276.

Badrane, H. and Tordo, N., 2001. Host switching in Lyssavirus history from the chiroptera to the carnivora orders. J. Virol., 75, 8096-80104.

Gould, R. A., Hyatt, A. D., Lunt, R., Kattenbelt, A. J., Hengstberger, S. and Blacksell .S.D., 1998. Characterization of a novel Lyssavirus isolated from Pteropid bats in Australia. Virus Res., 54, 165-187.

Ito, H., Minamoto, N., Watanabe, T., Goto, H., Luo, T. R., Sugiyama, M., Kinjo, T., Mannen, K., Mifune, K., Konobe, T., Yoshida, I. and Takamizawa, A., 1994. A unique mutation of glycoprotein gene of the attenuated RC-HL strain of rabies virus, a seed virus used for production of animal vaccine in Japan. Microbiol. Immunol., 38, 479-482.

Luo, T. R., Minamoto, N., Hishida, M., Yamamoto, K., Fujise, T., Hiraga, S., Ito, N., Sugiyama, M. and Kinjo, T., 1998. Antigenic and functional analyses of glycoprotein of rabies virus using monoclonal antibodies. Microbiol. Immunol., 42, 187-193.

Mebatsion, T., Weiland, F. and Conzelmann, K. K., 1999. Matrix protein of rabies virus is responsible for the assembly and budding of bullet-shaped particle and interacts with the transmembrane spike glycoprotein. J. Virol., 73, 242-250.

Morimoto, K., Hooper, C., Spitsin, S., Koprowski, H. and Dietzschold, B., 1999. Pathogenicity of different Rabies variants inversely correlates with apoptosis and rabies virus glycoprotein expression in infected primary neuron culture. J. Virol., 73, 510 - 518.

Saitou, N. and Nei, M., 1987. The neighborjoining method: a new method for reconstructing phylogenetic trees. Mol. Biol. Evol., 4, 406-425.

Tang, Q., Orciari, L. A., Rupprechti, C. E. and Zhao, X., 2000. Sequencing and position analysis of the glycoprotein gene of four Chinese rabies viruses. Zhongguo Bingduxue, 15, 22-33.

Walker, P. J. and Kongsuwan, K., 1999. Deduced structural model for animal Rhabdovirus glycoproteins. J. Gen. Virol. 80, 1211-1220.

Wiktor, T., Gyorgy, E., Schlumberger, D., Sokol, F. and Koprowski, H., 1973. Antigenic properties of rabies virus component. J. Immunol. 110, 269-276. 